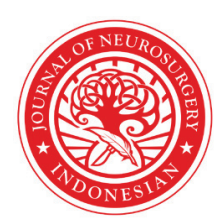

\title{
Unusual sized posterior neck tumor found in a child: A case report
}

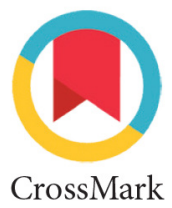

\author{
Yee Hwa Khoo ${ }^{1 *}$, Nishanthi Apparow ${ }^{1}$, Ananda Arumugam¹
}

\section{ABSTRACT}

Introduction: Giant lipoma, especially in children, are rare, representing a real diagnostic and therapeutic challenge. Workup to establish the diagnosis is vital. The occurrence of such giant lipoma is rare as parents would seek medical attention in such cases.

Case presentation: This is a case report of a healthy five years old boy, presented with swelling over the posterior neck since he was one year old. Initially, the swelling was the size of a golf ball. In time, the soft tissue swelling increased in size over a year. Clinically, the child was not in any form of pain and distress; neither was he fretful. Vital signs were typical, and he was active as usual. The swelling was not accompanied by any pain, abnormal discharge, or restricted neck movement. On examination, there was a solitary lesion measuring $30 \times 32 \times 33 \mathrm{~cm}$ on the posterior aspect of the child's neck. There was a small pressure ulcer noted over the middle part of the mass. However, no discharge was noted from the pustule. Magnetic resonance imaging of the neck was done and was reported as a soft tissue tumor with no intracranial or intraspinal involvement. The patient underwent operation under general anesthesia and excision of lipoma. Histopathological investigation of the lesion came back as fibro lipoma. At the sixth month follow up, the child was well without any complications, and the wound was well healed with primary healing.

Conclusion: Lipomas of the head and neck in children can behave aggressively. CT and FNA can be ineffective in establishing a definitive diagnosis of a lipoma. MRI is the imaging modality of choice in defining lipomas of the head and neck. Resection of the mass if possible, will minimize the possibility of recurrence and also definitively exclude malignancy, such as lipoblastoma.

Keywords: Giant lipoma, neurosurgery, pediatrics, posterior neck lipoma

Cite This Article: Khoo, Y.H., Apparow, N., Arumugam, A. 2020. Unusual sized posterior neck tumor found in a child: A case report. Indonesian Journal of Neurosurgery 3(2): 38-40. D0I: 10.15562/ijn.v3i2.72

${ }^{1}$ Neurosurgical Department, Sabah Women and Children Hospital Likas, Sabah
*Corresponding author: Yee Hwa Khoo; Neurosurgical Department, Sabah Women and Children Hospital Likas, Sabah; khooyeehwa@gmail.com
Received: 2019-06-25

Accepted: 2019-12-05 Published: 2020-08-01

\section{INTRODUCTION}

Lipoma is a benign mesenchymal tumor occurring throughout the body. A giant lipoma as attributed by Sanchez et al as a soft tissue lesion that either measures at least $10 \mathrm{~cm}$ in any one dimension or weighs a minimum of 1000 gram..$^{1,2}$ Lipomatous tumors are rare in the head and neck region. Giant lipoma in children are rare, and diagnosing such condition in the pediatric age group poses as a challenge. Workup to establish the diagnosis is vital. The occurrence of these cases is minimal as in the pediatric age group, parents are vigilant towards the medical needs of their young and would seek medical attention early in such cases. Significant medical neglect, lack of insight, and reduced logistics give rise to such progress of the medical condition as such. Clinicians should ensure adequate preoperative diagnosis to assure adequate tumor excision as well as the optimal functional and cosmetic outcome. Their biological variant includes absolutely benign lesion to histologically benign but locally infiltrative and the notorious form of invasive type with metastatic potential. Surgical excision of a lipoma is often used as the definitive treatment modality.

\section{CASE PRESENTATION}

This is a case report of a five years old boy presented with swelling over the posterior neck since he was one year old. Initially, the swelling was merely the size of a golf ball. In time, the soft tissue swelling increased in size for a year. Because of the slow-growing rate of the swelling, logistic restrictions, and the benign nature of the swelling, the mother decided to opt for conservative management and observe the child at home. Clinically, the child was not in any form of pain and distress; neither was he fretful. The child is active and cheerful, developmental up to age, and had no systemic symptoms. The swelling was not accompanied by any pain, abnormal discharge, or restricted neck movement. On examination, there was a solitary lesion measuring $30 \times 32 \times 33 \mathrm{~cm}$ on the posterior aspect of the child's neck (Figure 1 and 2). There was a small pressure ulcer noted over the middle part of the mass. However, no discharge was noted from the pustule. The patient had no neurological deficit. Magnetic resonance imaging of the neck was done and was reported as a soft 
tissue tumor with no intracranial or intraspinal involvement. The lesion shows a high signal on $\mathrm{T} 1$ and saturates on fat-saturated sequences with minimal enhancement. T2 images show a signal on FSE T2 (Figure 3 and 4).

The patient underwent excision of lipoma in

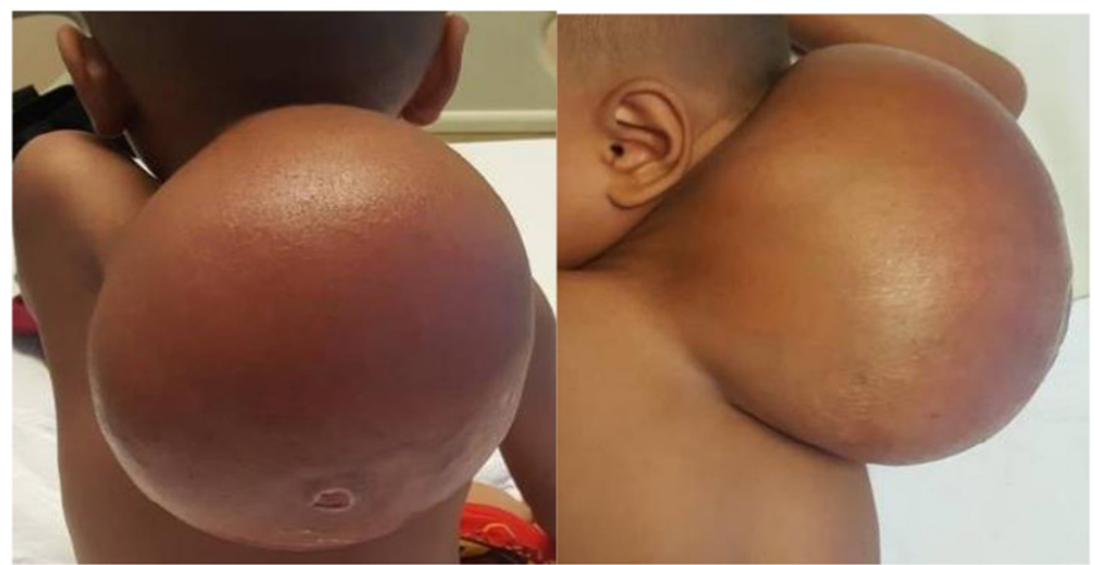

Figure 1 and 2. Solitary mass over the posterior neck with size $30 \mathrm{~cm}$ x 32 $\mathrm{cm} \times 33 \mathrm{~cm}$

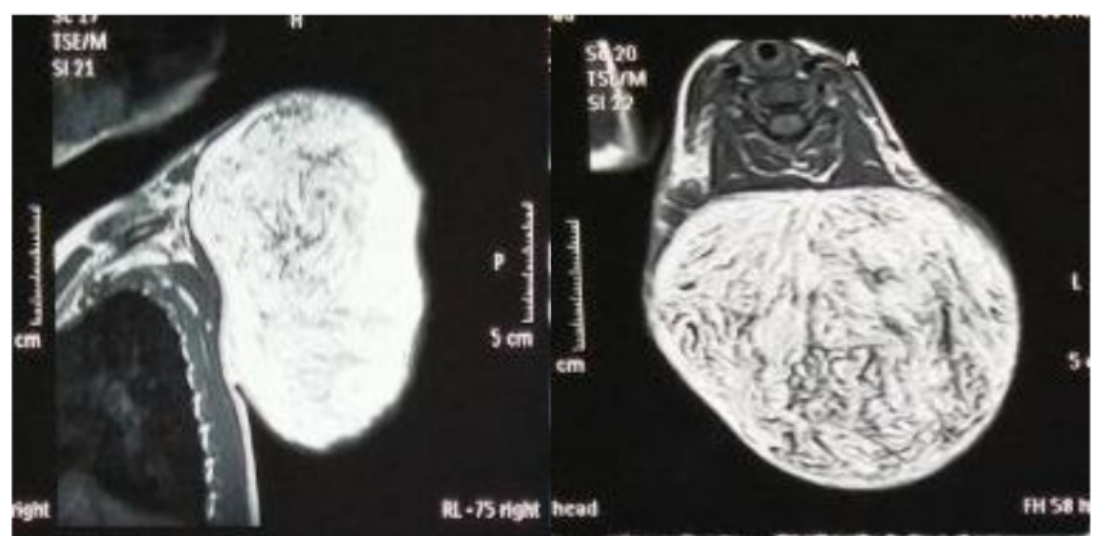

Figure 3 and 4. MRI of the neck reveals soft tissue tumors with no intracranial or intraspinal involvement.

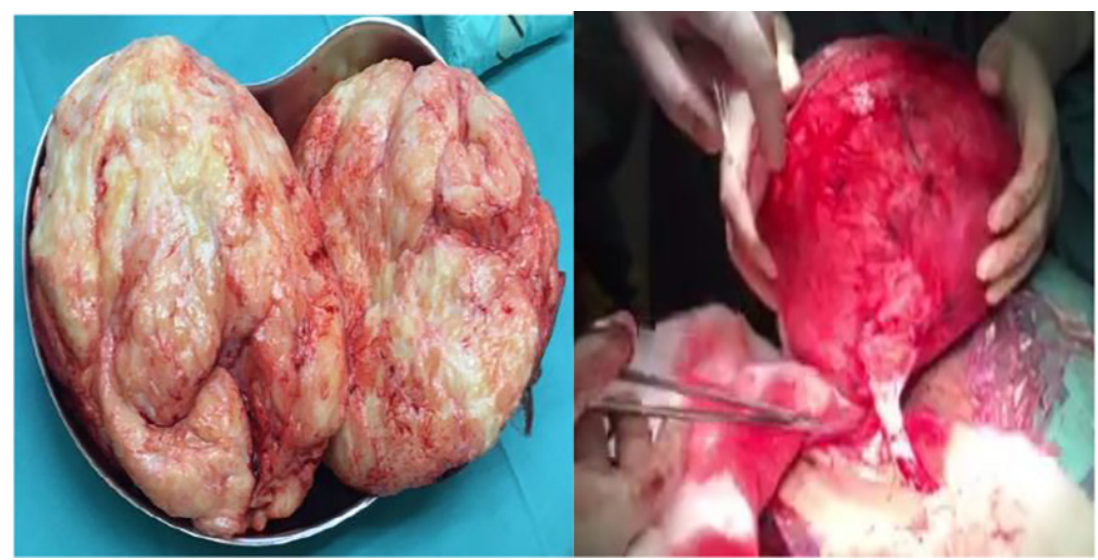

Figure 5 and 6. Excision of the lesion, yellowish fatty tissue on intraoperative finding the operating theatre. After intubation with general anesthesia, the patient was positioned in a prone position. At around base of lump, an elliptical incision was done. A superior and inferior skin flap is separated. Separation of lipoma inferiorly from surrounding tissues was easy and was performed with blunt dissection with Metzenbaum scissors. Vessels were coagulated with bipolar cautery for hemostasis and to separate the lipoma. The excess skin was cut, subcutaneous and skin layer closed separately after securing hemostasis and washout (Figure 5 and 6).

The resected mass weighs $2 \mathrm{~kg}$. Histopathology of the resected mass showed a well-encapsulated tumor composed of adipocytes in sheets separated by fibrovascular septa into lobular architecture. The adipocytes exhibit eccentrically located bland nuclei. No malignancy was seen, and it was diagnosed as fibrolipoma (Figure 7 and 8). The mass excised was weighed and noted to weigh an astounding 2020 grams. The surgical wound was closed in layers, and excess soft tissue refashioned for proper wound closure.

\section{DISCUSSION}

Lipoma occurs in almost all parts of the body where fat exists typically. It rarely occurs in children. Lipoma is typically a slow-growing tumor, only a few grow to a huge size. Only approximately $25 \%$ of the lipomas and their variants arise in the head and neck region. ${ }^{3}$ For the last decade, some of the English language literature noted that giant lipoma was reported found on the back and posterior cervical area. However, they all occur in adult ages. This case is quite rare because it occurred in a five years old child, and weigh 2020 grams, though lipoma in this region commonly occurs at the age of 40 to 60 years old. Radiological imaging such as computed tomography or magnetic resonance imaging helped with the diagnosis of unusual neck masses. The success of MRI in identifying well-differentiated lipomas among other fatty masses was as follows: sensitivity, $100 \%$; specificity, $83 \%$; accuracy, $84 \%$; positive predictive value, $38 \%$; and negative predictive value, $100 \%$. MRI is $100 \%$ specific in the diagnosis of simple lipoma. Sixty-three percent of lesions considered suspicious for well-differentiated liposarcoma were simple lipomas (13\%) and benign lipoma variants (50\%), including chondroid lipoma (13\%), osteolipoma (6\%), hibernoma (6\%), lipoleiomyoma (6\%), angiolipoma (6\%), and infarcted lipoma (13\%). Therefore the diagnosis of this patient was made at the imaging stage and further confirmed by histopathological evidence. Preoperative diagnosis is to assure adequate tumor excision as well as the optimal functional and 


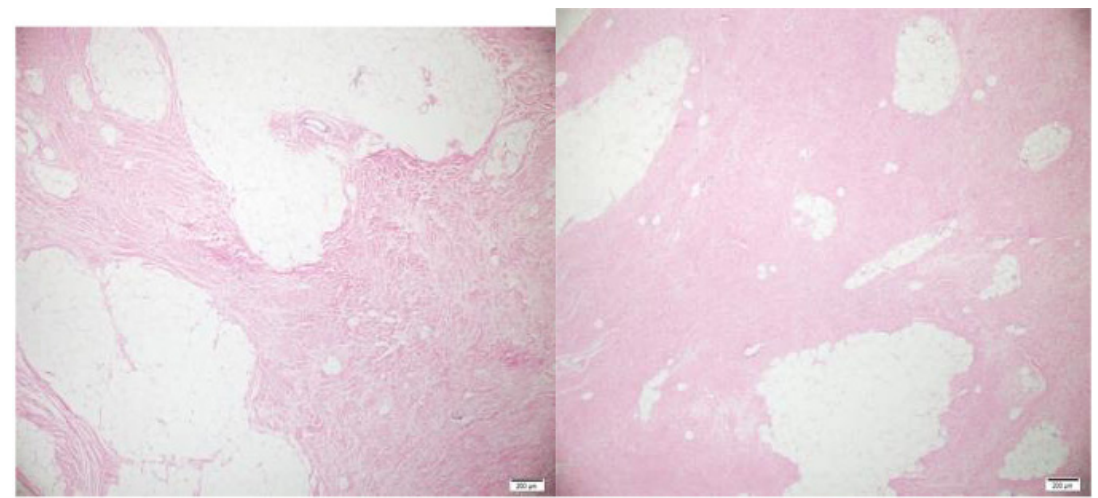

Figure 7 and 8. HPE examination

cosmetic outcome. Surgical management was the modality of the choice of treatment on this patient, and intraoperatively tumor was easily excised, and we removed it in one block.

There was no excessive bleeding during the surgery as we managed to separate the tumors around the pseudo-capsule and cauterized the feeding arteries. The choice of treatment includes complete excision since they have a well-defined pseudo capsule, dissection around it is performed smoothly. Liposuction, as another choice of treatment, has also been reported. ${ }^{4}$ Liposuction is sometimes preferred to excision because it causes less scarring, but the recurrence rate may be higher compared to excision if the residual tumor, including the capsule remaining postoperatively..$^{5}$ Rarely, lipoma transforms into malignancy. However, the possibility of malignancy, and increased mitotic rate, the occurrence of liposarcoma, cannot be taken lightly. ${ }^{6}$

\section{CONCLUSION}

Lipomas of the head and neck in children can behave aggressively. CT and FNA can be ineffective in establishing a definitive diagnosis of a lipoma.
MRI is the imaging modality of choice in defining lipomas of the head and neck. Resection of the mass, if possible, will minimize the possibility of recurrence and also definitively exclude malignancy, such as lipoblastoma.

\section{CONFLICT OF INTEREST}

There is no potential conflict of interest relevant to this article reported.

\section{FUNDING}

No specific funding was provided for this article.

\section{AUTHOR'S CONTRIBUTION}

Authors' contributions include manuscript preparation, literature search, manuscript editing and final manuscript.

\section{References}

1. Waqar SH, Abdullah MT, Khan SA, Malik ZI, Zahid MA. Giant lipoma of the thigh. Ann Pak Inst Med Sci. 2014; 10(1) $57-59$.

2. E. Aydo_gdu, S. Yýldýrým, G. Eker, T. Ak€oz. Giant lipoma of the back. Dermatol Surg. 2004; 30(1): 121 - 122.

3. Kransdorf MJ. Benign soft-tissue tumors in a large referral population: Distribution of specific diagnoses by age, sex, and location. AJR Am J Roentgenol. 1995; 164: 395 - 402.

4. Gaskin CM, Helms CA. Lipomas, lipoma variants and well-differentiated liposarcomas (atypical lipomas): Results of MRI evaluations of 126 consecutive fatty masses. AJR Am J Roentgenol. 2004; 182(3): 733 - 9. DOI: 10.2214/ ajr.182.3.1820733.

5. Rubenstein R, Roenigk HH, Garden JM, Goldberg NS, Pinski JB. Liposuction for lipomas. J Dermatol Surg Oncol. 1985; $11: 1070-4$.

6. Casani AP, Marchetti M, Dallan I, Cagno MC, Berretini S. Liposarcoma of the cervico-nuchal region. Otolaryngol Head Neck Surg. 2005; 133: 1 - 3.

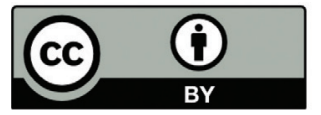

This work is licensed under a Creative Commons Attribution 